\title{
Synthesis of High Aspect-Ratio Gold Nanowires with Highly Porous Morphology
}

\author{
Lucas P. Johnson and Janis G. Matisons \\ School of Chemical and Physical Sciences, Flinders University, Adelaide, SA 5001, Australia \\ Correspondence should be addressed to Lucas P. Johnson, lucas.johnson@flinders.edu.au
}

Received 4 April 2012; Accepted 8 May 2012

Academic Editors: S.-H. Kim, W. Lee, and M. Mirzaei

Copyright ( $) 2012$ L. P. Johnson and J. G. Matisons. This is an open access article distributed under the Creative Commons Attribution License, which permits unrestricted use, distribution, and reproduction in any medium, provided the original work is properly cited.

\begin{abstract}
Here, we demonstrate the formation of porous gold nanowires with diameters $<60 \mathrm{~nm}$ by a two-step process involving the successive electrodeposition of silver then gold into the pores of a track-etched polycarbonate filtration membrane, followed by treatment with nitric acid. The resulting nanowires possess a unique, highly porous morphology, which yields a very high accessible surface area to volume ratio compared to solid nanowires of the same dimensions. Combined with the high aspect ratio of these particles (which allows for easy isolation from solution), this makes them eminently suitable for use in catalysis and sensing applications. The formation of such porous gold nanostructures was ascertained to result from the low diffusivity of the silver species within the narrow membrane pores.
\end{abstract}

\section{Introduction}

Gold nanoparticles, discrete ensembles of gold atoms with one or more dimensions on the submicron scale, are materials that have attracted considerable interest in recent decades owing to their unique-size-dependant electronic and optical properties as well as their high unit surface areas $[1,2]$; the combination of which makes them particularly suited to use in a variety of catalytic [3-9] and chemical sensor applications [10-15].

Typically, these applications utilize small solid nanoparticles ranging between $2-50 \mathrm{~nm}$ in diameter, as this size range is where such nanoparticles exhibit their useful properties $[3-5,16-21]$. However, the use of such small nanoparticles presents a number of difficulties. For instance, gold nanoparticles with these dimensions possess rather high surface energies [22]. Therefore, in order to maintain a high unit surface area, the implementation of methods to stabilize them against aggregation is required [23, 24]. These methods must be carefully selected so that useful (in particular surface) properties are retained $[25,26]$. Such small nanoparticles are also relatively difficult to separate from solution, making their reuse in applications costly.
One recent development that promises to help overcome these issues has been the preparation of porous gold nanowires. Owing to their high aspect (length to diameter) ratios, such particles can be easily isolated from solution, while their porosity yields exposed surface features (known as ligaments) with dimensions suitable for use in applications, even when the particles as a whole are relatively large [27-30]. Such porosity also ensures that these particles have much higher surface areas relative to solid nanowires with equivalent dimensions. Furthermore, unlike solid nanoparticles, aggregation is not a significant problem, as the particle ligaments and surfaces are largely internal and, therefore, remain accessible following aggregation.

Very few methods have been published to date by which porous gold nanowires can be synthesized. Current methods involve the electrodeposition of gold into a nanoporous template; either as a pure gold, followed by galvanic exchange reaction $[31,32]$, or as a single-phase, two-component gold alloy, which is subsequently dealloyed through chemical etching of the nongold metal [33-39]. Of these methods, electrodeposition of gold alloys into nanoporous templates followed by selective chemical etching is perhaps the most advantageous. It is a relatively simple synthetic technique 


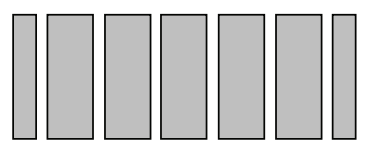

(a)

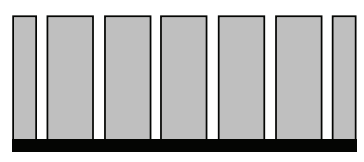

(b)

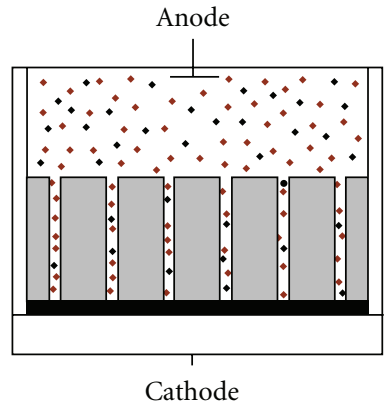

(c)

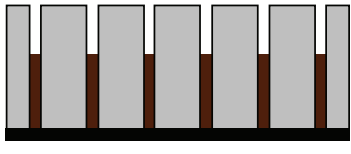

(d)

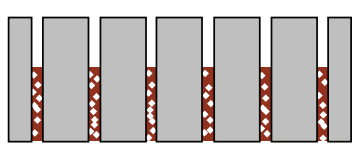

(e)

FIGURE 1: The process for synthesizing porous gold nanowires by template electrodeposition with chemical etching: a nanoporous template (a) is coated on one side with a conductive layer (b). Gold and the sacrificial metal are then simultaneously electroplated exclusively into the template pores onto the conductive layer $(\mathrm{c})$. The resulting alloy filled template $(\mathrm{d})$ is then treated to de-alloy the nanowires (and thereby form pores) (e), followed by removal of the template to isolate the nanowires.

(as depicted in Figure 1) that is amenable to the formation of large quantities of nanoparticles, and it provides a high level of control over the particle dimensions (through choice of template pore diameter and the quantity of deposited metal) as well as morphology and composition (through modulation of various deposition parameters and the use of multiple plating steps to incorporate different components, both porous and solid, along the length of the particles) [4048].

To date, however, the vast majority of the literature has only involved large porous nanowires ( $\geq 200 \mathrm{~nm}$ in diameter) [33-38], which are not ideal for use in the aforementioned applications as they suffer from either comparatively small surface areas (in the case of wires with large pores) or relatively hard to access internal surfaces due to the large diameter of these nanowires (in the case of particles with small pores). Nanowires with smaller diameters would help remedy these problems; however, the only report to date of such narrow $(<60 \mathrm{~nm}$ diameter) porous gold nanowires involve particles with what has been described as a "hinged" morphology, the formation of which has been ascribed to the limits placed on the nanostructure growth mechanism (spinodal decomposition of the deposited gold upon etching of the silver [49-51]) by the narrow membrane pores [39]. Porous gold nanowires with this morphology are characterized by large ligaments (relative to the nanowire diameter) and correspondingly small pores, making these particles nonideal for use in catalytic and sensing applications using gold.

Here, we report the synthesis of narrow (diameter $\leq 60 \mathrm{~nm}$ ), high-aspect-ratio porous gold nanowires (through electrodeposition into nanoporous templates) which possess a unique porous morphology that is characterized by small ligament dimensions. These nanowires were unexpectedly fabricated using a modified version of an established procedure [33], involving the electrodeposition of silver and gold within the narrow pores of a commercially available filtration membrane in discrete steps, rather than through the simultaneous electrodeposition of both metals from a single plating solution. This approach is advantageous not only in that it yields highly porous gold nanowires with an open pore structure, but also in that it grants a larger degree of freedom in the choice of electroplating bath chemistries: a feature that is not present in current literature methods, which require the metal species to have similar reduction potentials [3338 .

\section{Experimental Section}

2.1. Nanorod Synthesis. A commercially available radiation track-etched, hydrophilic polycarbonate membrane (Sterlitech, $47 \mathrm{~mm}$ diameter, $>5 \mu \mathrm{m}$ thickness, $10 \mathrm{~nm}$ nominal pore diameter) is first prepared for use as a nanoporous template by sputter-coating one side with a $10 \mathrm{~nm}$ thick layer of chromium (adhesion layer) followed by a $200 \mathrm{~nm}$ thick layer of silver (conducting layer) using a Cressington 208HR sputter coater. The silver-coated membrane is then placed coated side down onto the cathode; a steel contact plate (adhesion of the membrane to the plate is enhanced by placing 1-2 drops of water onto the contact plate), and the electrochemical cell is assembled by clamping a rubber O-ring ( $34 \mathrm{~mm}$ diameter) and glass tube onto the membrane (as depicted in Figure 2). This setup ensures that metal deposition occurs exclusively into the pores of the membrane. The cell is then filled with Millipore Nanopure water $(18 \mathrm{M} \Omega)$ and the membrane left to soak for $30 \mathrm{~min}$ in order to completely wet the membrane pores so subsequent electrodeposition will occur in all pores simultaneously [52, 53].

Following this, the water is replaced with a silver electroplating solution (Silver Cyless II (silver oxide), Technic). $\mathrm{An} \mathrm{Ag} / \mathrm{AgCl}$ reference electrode and a platinum counter electrode (anodic surface area $\sim 2 \mathrm{~cm}^{2}$ ) are then suspended in the solution close to the membrane. $100 \mathrm{mC}$ of silver $(0.112 \mathrm{mg})$ is then deposited potentiostatically $(-1.0 \mathrm{~V}$ versus $\mathrm{Ag} / \mathrm{AgCl}$ ) using a BAS $100 \mathrm{~b}$ electrochemical analyzer. Following silver deposition, the cell is thoroughly rinsed three times with nanopure water, and the cell is then filled with a gold plating solution (434 neutral soft gold (gold cyanide), Technic). This electrodeposition and rinsing process is then carried out with the gold solution in the same manner. 


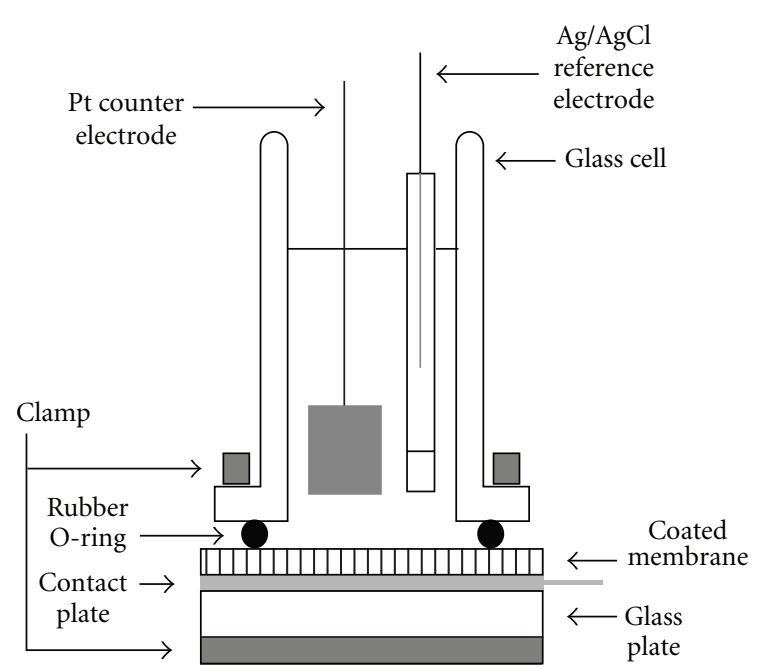

Figure 2: Diagram of the electroplating cell used to deposit metal into the pores of a nanoporous membrane.

2.2. Nanowire Collection. The filled membrane is first subjected to a brief $(10 \mathrm{sec})$ treatment with $4 \mathrm{M}$ nitric acid at room temperature in order to selectively dissolve the silver. The membrane is then rinsed with distilled water, blotted dry, and placed into a $10 \mathrm{~mL}$ polyethylene centrifuge tube. The tube is then filled with $6 \mathrm{~mL}$ of dichloromethane and the membrane is dissolved with brief sonication and shaking. The nanowires are then sedimented into a pellet by centrifuging the suspension at 3200 RPM (1090 g) for $30 \mathrm{~min}$. The supernatant is removed and replaced with $3 \mathrm{~mL}$ of pure dichloromethane and the pellet is broken up with sonication. This process is repeated several times to remove the residual polymer from the membrane. This process is then further repeated twice more using ethanol instead of dichloromethane, with the final product being stored in $3 \mathrm{~mL}$ of ethanol.

2.3. Nanorod Characterisation. Nanowire samples are imaged by transmission electron microscopy (TEM) using a Phillips CM200 TEM equipped with a Gatan 832 SC1000 CCD camera and energy-dispersive X-ray analysis (EDAX) apparatus at an accelerating voltage of $200 \mathrm{kV}$. The spot size setting for general imaging is 3 , but it is set as small as spot size 7 for collecting elemental composition (EDAX) data at specific locations of the nanowires. Nanowire samples are prepared for TEM by briefly sonicating nanowire suspensions in ethanol, and then placing one drop $(\sim 5 \mu \mathrm{L})$ of the resulting suspension onto a $\mathrm{Cu}$ grid (200 mesh) that is coated with a formvar membrane. The ethanol is then allowed to evaporate before collecting images.

\section{Results}

TEM images (along with EDAX analysis) of the nanowires that result from the deposition of $25 \mathrm{mC}$ of gold $(0.051 \mathrm{mg})$ into the pores of polycarbonate membranes under the conditions used in this work are shown in Figures 3 and 4, respectively. It is observed that highly porous nanowires
(Figure 3) that are comprised entirely of gold (Figure 4) have formed. These nanowires have a mean diameter of $52.4 \mathrm{~nm}$ (standard deviation: $6.3 \mathrm{~nm}$ ); wider than the rated $10 \mathrm{~nm}$ pore diameter of these nanoporous templates due to the wellknown "cigar shaped" pores of commercially available PCTE membranes [53-55]. They are also noted to be substantially longer than their diameter (a relatively high aspect ratio).

Examination of the fine structure of these nanowires by high-resolution TEM (Figures 5 and 6) shows that they possess a structural morphology that is markedly different from the so called "hinged" morphology that is characteristic of similarly sized porous gold nanowires as previously cited in the literature [39]. While the hinged morphology is comprised of stacks of large ligaments (relative to the nanowire diameter) that are aligned perpendicular to the length of the nanowires (giving them a lumpy appearance), the nanowires produced in this work possess a porous structure that is defined by relatively small ligaments (most often aligned either parallel or perpendicular to the length of the nanowires), with large open pores extending through the width of the nanowires.

The formation of such high-porosity gold nanowires, while an improvement over previous work, is noted as being most unusual, as the procedure used in their synthesis involves the deposition of gold under electroplating conditions typically used to form solid gold nanowires rather than porous gold $[53,54,56-60]$. In order to explain this result, several possibilities were considered.

Firstly, it was considered that the current density during electrodeposition of the gold may have been high enough that hydrogen gas was evolved at the cathode during the electrodeposition process, with the resulting bubbles remaining trapped in the narrow membrane pores, thereby creating voids in the deposited gold [61].

To determine if this may be the case, current versus time data for the gold deposition was thus examined (Figure 7). As can be seen, there is an initial peak in current which rapidly drops, followed by a more gradual decrease toward a steady state. The initial current spike is ascribed to rapid charging of the cathode electric double layer upon application of the nonequilibrium potential difference between the anode and cathode, as the increase in potential difference leads to an increase in the charge separation in the diffuse double layer; the movement of these charges being experienced as a (capacitive) current $[62,63]$. This charging process is completed rapidly, yielding the drop in current to a level commensurate with that due to the electrodeposition process. The subsequent gradual current decrease toward a steady value $\left(2.98 \mathrm{~mA} / \mathrm{cm}^{2}\right)$ is indicative of the formation of a diffusion layer of growth species within the pores; a result of the rate of reduction of metal species at this overpotential being greater than the rate of replenishment of the metal species near the cathode by mass transport into the pores from the bulk solution. This then suggests that the deposition process is mass transport controlled, which is not unexpected given the membrane pore dimensions and negative charge on the gold species [62]. Importantly, it can be seen from this data that the actual current density at the cathode (excluding the capacitive "current") is well within 


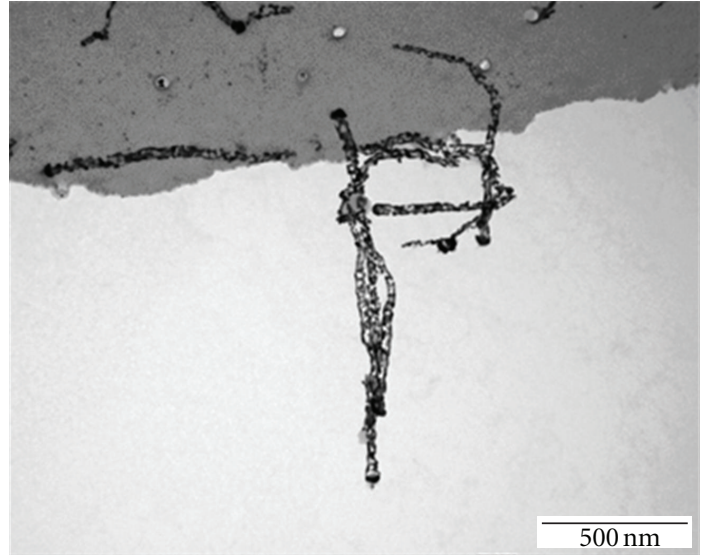

(a)

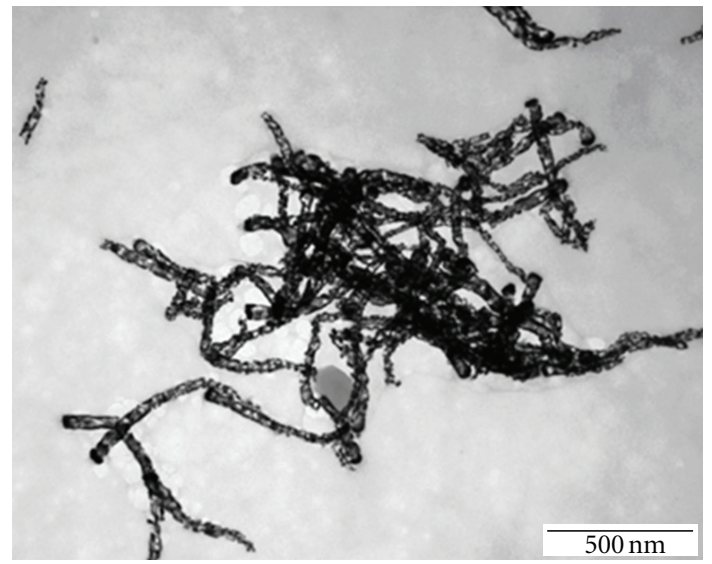

(b)

FIGURE 3: TEM images of porous gold nanowires ( $25 \mathrm{mC})$.

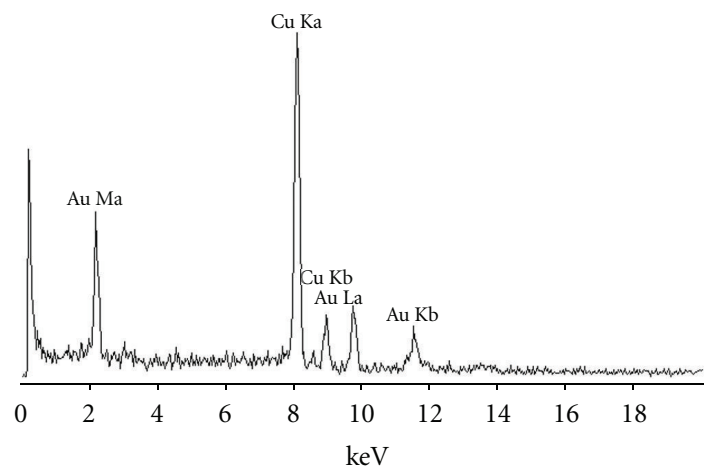

FIgURE 4: EDAX analysis of porous gold nanowires. The copper signal is attributed to background scattering from the copper TEM grid.

the limits for gold deposition with high current efficiency for this plating solution $\left(1.1-10.8 \mathrm{~mA} / \mathrm{cm}^{2}\right)$ for effectively the whole deposition period [64]. This means that essentially all of the charge passed was used to reduce the gold species rather than evolve hydrogen gas.

A second possible explanation is that these structures are in fact incompletely formed gold nanotubes, which

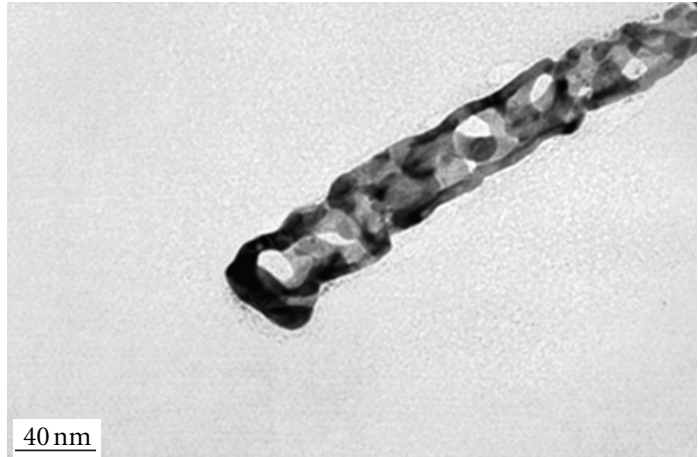

FIGURE 5: High-resolution TEM image of porous gold nanowires.

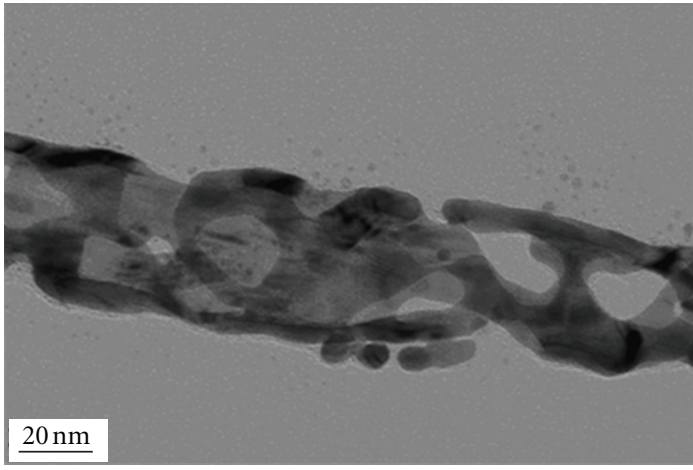

FIGURE 6: High-resolution TEM image of porous gold nanowires that demonstrates their unique morphology.

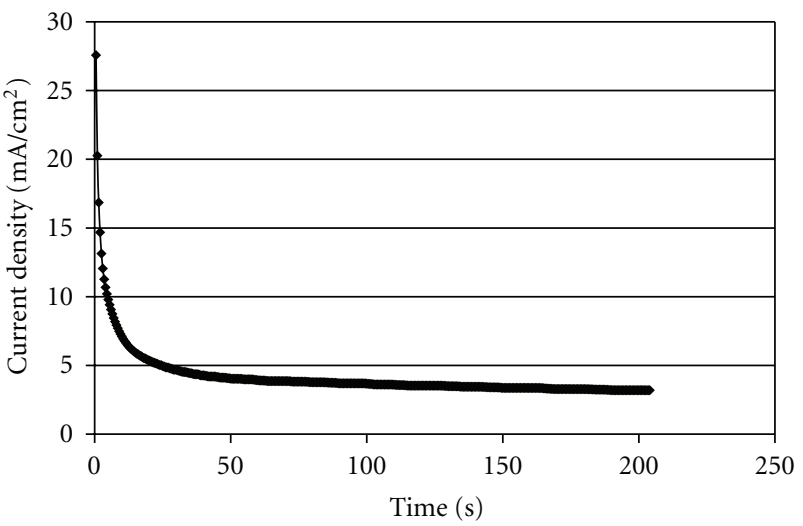

FIGURE 7: Current versus time data for the electrodeposition of gold into nanoporous templates.

can come about due to the reduction of gold species that are preferentially adsorbed on the walls of the membrane pores $[61,65]$. This explanation was contemplated as the prevailing conditions during the gold deposition; namely, low overpotentials (the reduction potential of gold cyanide is $-0.6 \mathrm{~V}$ versus $\mathrm{Ag} / \mathrm{AgCl}[66]$ ) and a low gold concentration near the cathode (current versus time data in Figure 7 indicates diffusion limited growth) are conducive to the formation of such gold structures [61]. In order to test this hypothesis, syntheses were carried out with larger amounts 

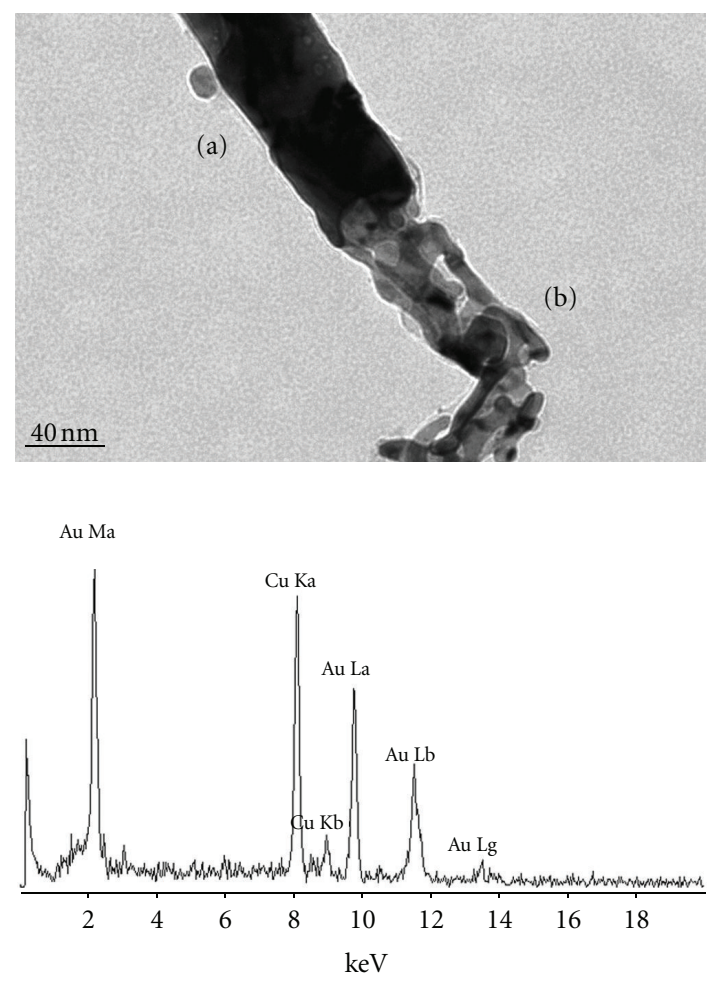

(a)

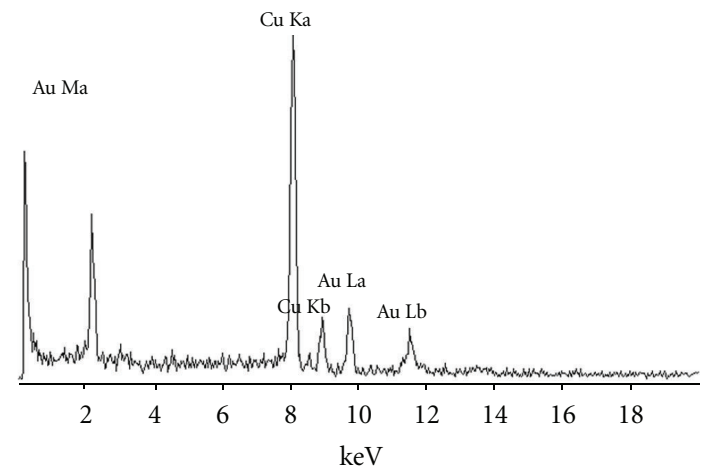

(b)

FIGURE 8: High-resolution TEM image and EDAX analysis of a bisegmented nanowire comprised of (a) a solid gold segment and (b) a porous gold segment.

of gold being deposited into the membrane pores (50, 75, and $100 \mathrm{mC}$ ). The reason for doing this is that the growth mechanism of these structures involves the formation of long complete nanotubes followed by inward growth toward solid nanowires (starting at the cathode surface and advancing towards the pore entrance). Therefore, greater amounts of deposited gold should first yield complete nanotubes, followed by thicker nanotubes that ultimately grow into solid nanowires [61]. However, TEM imaging (Figure 8) shows that depositing more gold instead yields nanowires that retain a porous gold segment (similar to those produced after the deposition of $25 \mathrm{mC}$ of gold) attached to a solid segment comprised of gold, which gets progressively longer with increased quantities of deposited gold (Figures 9(a), 9(b), and 9(c)).

Based on this result, it was then hypothesized that these porous gold structures may in fact be due to codeposition of the gold with residual silver species from the previous electrodeposition, which remain within the pores even following the use of established pore rinsing methods. This explanation would account for the formation of both the porous segments (coarsening of the gold upon chemical removal of the silver from the resulting gold-silver alloy) and the additional solid gold segments upon further gold deposition (residual silver species are eventually depleted yielding only gold deposition). To test this explanation, TEM images were collected of nanowires ( $50 \mathrm{mC}$ of charge passed) prior to treatment with nitric acid (Figure 10). As predicted, nanowires with two distinct segments are present; a solid gold segment (Figure 10(a)), and a lower electron density segment that is confirmed by EDAX to be comprised of a mixture of both gold and silver (Figure 10(b)).

Further tests were then performed to determine the source of the residual silver species. These tests, deposition of gold preceded by rinsing of the membrane pores between depositions by sonication or by the application of a electric potential, were designed to discriminate between the two most likely candidates: (a) silver species being generated upon introduction of the gold solution to the cell, that is, dissolution/displacement of the deposited silver by the gold cyanide solution $\left(\mathrm{Ag} \rightarrow \mathrm{Ag}(\mathrm{CN})_{2}{ }^{-}\right)$and (b) silver species remaining in the pores from the silver electrodeposition, that is, incomplete rinsing of the narrow membrane pores between electrodepositions when using the standard literature rinsing method (with the necessary conversion of silver oxide $\left(E_{\text {red }}: 0.34 \mathrm{~V}\right)$ to silver cyanide $\left(E_{\text {red }}:-0.31 \mathrm{~V}\right)$ by free cyanide species in the gold cyanide $\left(E_{\text {red }}:-0.6 \mathrm{~V}\right)$ solution in order for codeposition to occur [66]). As can be seen in Figure 11, sonication between depositions (three consecutive $5 \mathrm{~min}$ rinses in nanopure water under mild sonication) yielded gold nanowires consisting of a single solid segment, suggesting that silver species remaining in the pores from silver deposition is the cause of porous gold nanowire formation.

Likewise, rinsing the membrane pores between electrodeposition steps by applying a potential of $-1.0 \mathrm{~V}$ versus $\mathrm{Ag} / \mathrm{AgCl}$ (to reduce the residual silver species) in a $1 \mathrm{mM}$ $\mathrm{KOH}$ electrolyte solution (which acts to promote reduction of the residual silver oxide by ensuring it remains soluble and by weakening electro-osmosis effects in these polyvinylpyrrolidone coated pores [67]) results in solid gold nanowires with markedly reduced formation of porous segments (Figure 12), further supporting the argument that remnants of the silver plating solution are responsible for the formation of gold nanostructures.

Overall, given that gold-silver alloyed nanowires are formed by this procedure, and that porous gold nanostructures are formed following treatment with nitric acid, it is reasonable to conclude that these structures are a result of the coarsening of the gold during chemical removal of the silver. 

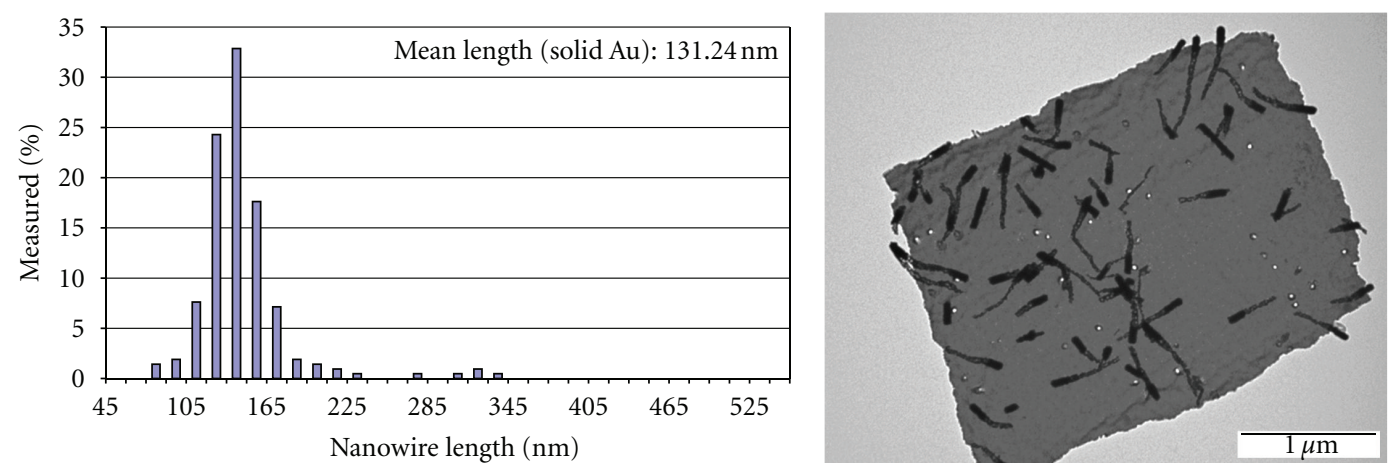

(a)
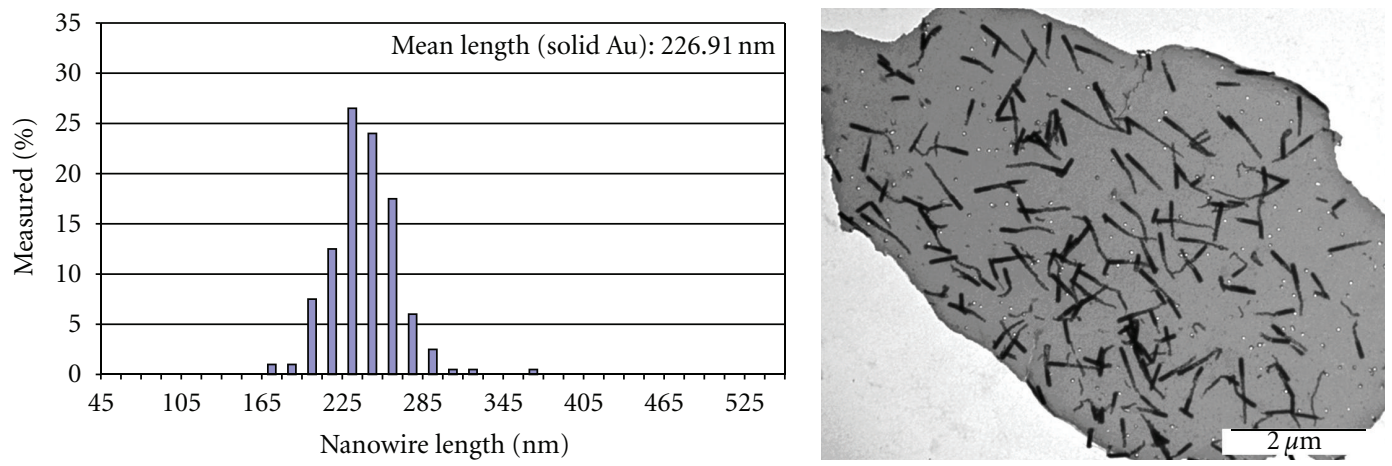

(b)
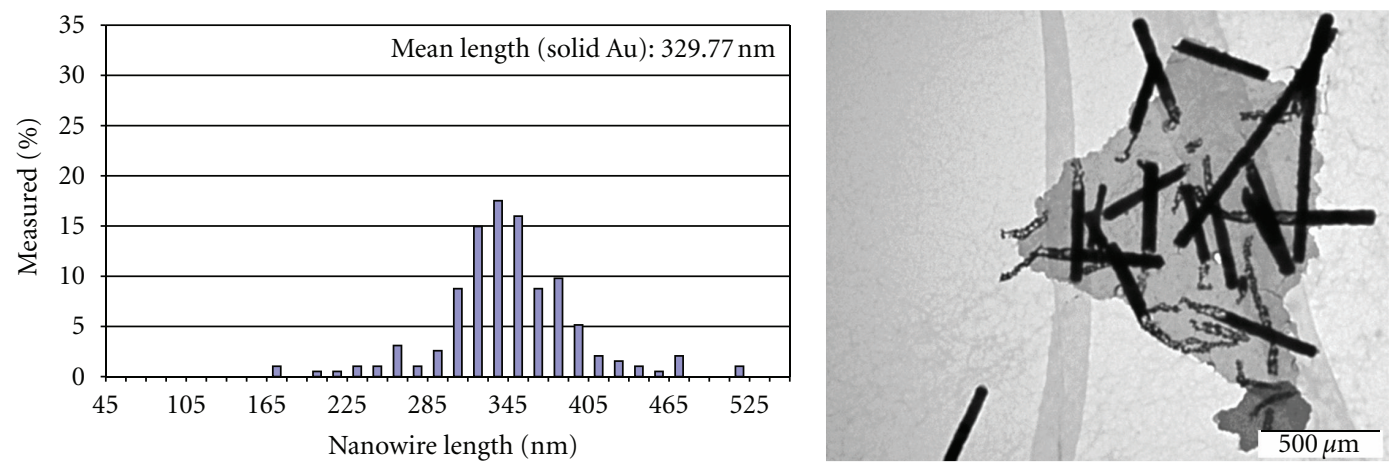

(c)

Figure 9: (a) Bisegmented gold nanowires resulting from 0.05 C of gold being deposited. (b) Bisegmented gold nanowires resulting from $0.075 \mathrm{C}$ of gold being deposited. (c) Bisegmented gold nanowires resulting from $0.1 \mathrm{C}$ of gold being deposited.

This result has interesting implications for the current understanding of how such structure form, as previous work on nanoporous gold nanowires established that a hinged morphology resulted due to confinement of the spinodal decomposition to narrow membrane pores [39]. However, despite the use of similar pore diameters in this work, markedly different nanowire morphologies were observed, which implies that there other factors that influence the nanostructure formation. While these factors were not directly elucidated here, they are likely related to the different processing conditions (lower acid concentration, short etching time, different electroplating solution composition, and membrane template) and even possibly the different synthesis conditions under which the gold-silver alloyed nanowires are formed in this work, as both sets of these factors influence the starting distribution of the gold atoms as well as the mobility of gold adatoms and surface energy of the gold clusters during the coarsening process $[49,68,69]$.

\section{Conclusions}

In summary, it was found that narrow, high-porosity gold nanowires result from the successive electrodeposition of silver and gold into the pores of commercially available track etched polycarbonate filtration membranes. This unexpected nanostructure formation was found to occur due to the entrapment of residual silver species within the narrow 

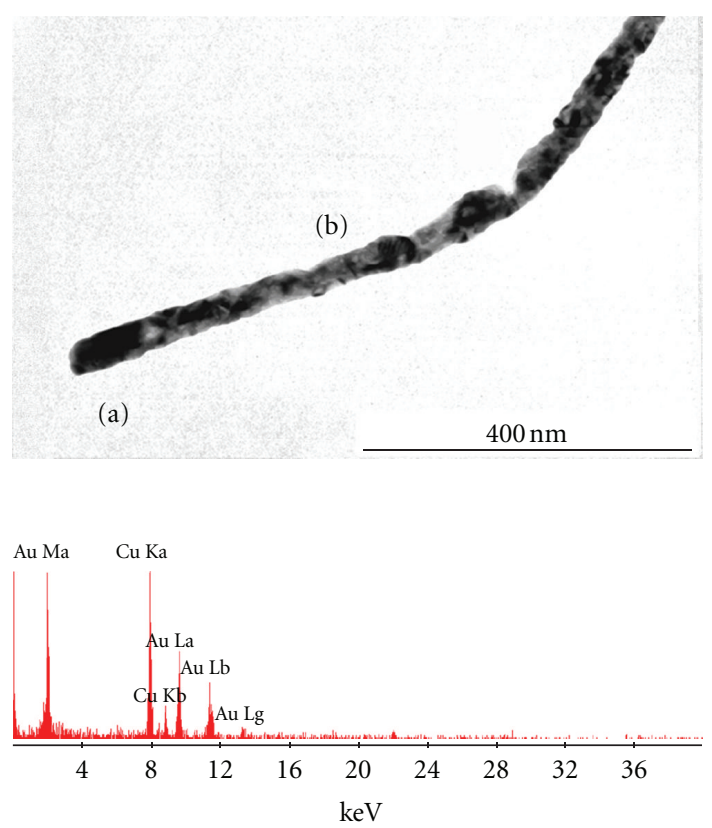

(a)

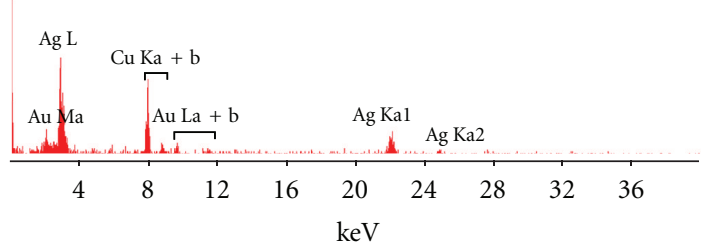

(b)

FIgURE 10: TEM image and EDAX analysis of a bisegmented nanowire prior to dealloying.

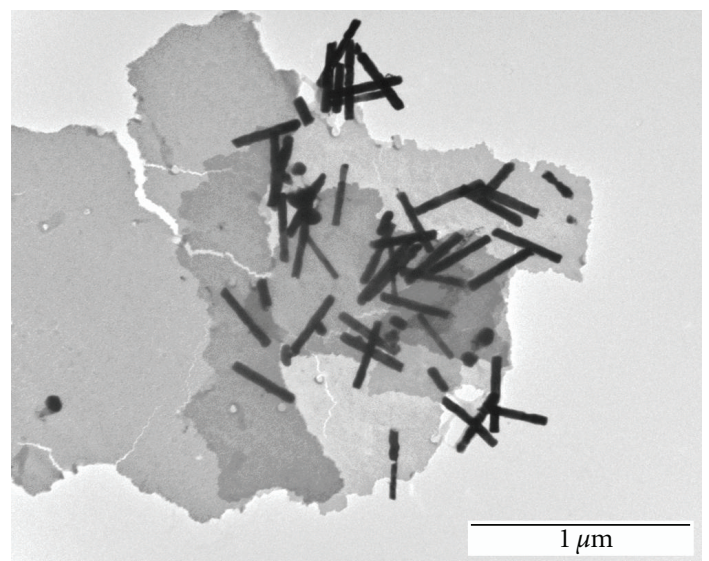

FIGURE 11: TEM image of gold nanowires synthesized with the membrane template being emptied of residual species between metal depositions by sonication.

membrane pores. This resulted in their codeposition with gold, with subsequent etching of the silver with nitric acid yielding porous gold through a well-known spinodal decomposition mechanism. The method described here for forming porous gold nanowires is advantageous in that it allows for the use of a broad range of plating solutions, while offering

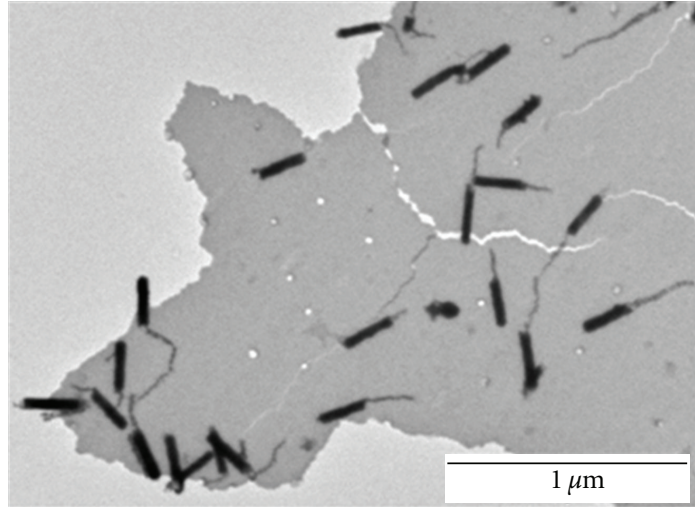

(a)

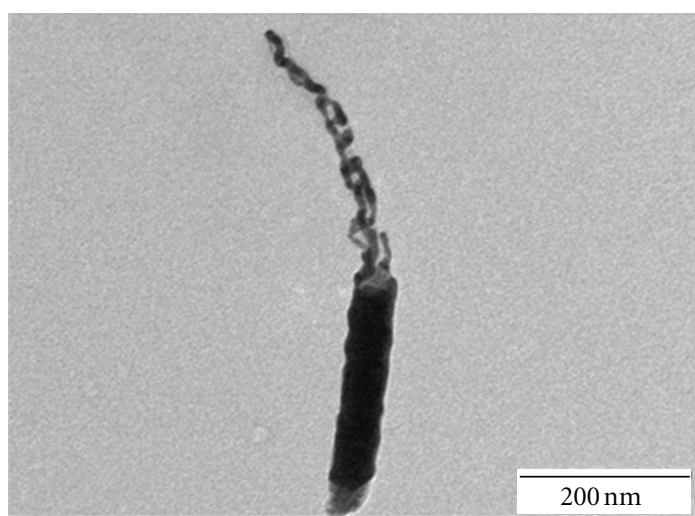

(b)

Figure 12: TEM image of gold nanowires synthesized with the membrane template being emptied of residual species between metal depositions by electrodeposition.

an improvement over previously produced porous gold nanowires due to their smaller diameters and ligament sizes, and, therefore, correspondingly higher accessible surface areas. Such nanostructures are anticipated to be useful in a wide range of catalytic and sensing applications.

\section{Acknowledgments}

The authors thank the Australian government for providing funding for this work under the Australian Postgraduate Award (APA), and Mr. Kerry Gascoigne for assistance with electron microscopy.

\section{References}

[1] J. Wang, R. Polsky, and D. Xu, "Silver-enhanced colloidal gold electrochemical stripping detection of DNA hybridization," Langmuir, vol. 17, no. 19, pp. 5739-5741, 2001.

[2] J. Wang, D. Xu, and R. Polsky, "Magnetically-induced solidstate electrochemical detection of DNA hybridization," Journal of the American Chemical Society, vol. 124, no. 16, pp. 42084209, 2002.

[3] D. Thompson, "New advances in gold catalysis part II," Gold Bulletin, vol. 32, no. 1, pp. 12-19, 1999. 
[4] D. Thompson, "New advances in gold catalysis part I," Gold Bulletin, vol. 31, no. 4, pp. 111-118, 1998.

[5] D. Andreeva, "Low temperature water gas shift over gold catalysts," Gold Bulletin, vol. 35, no. 3, pp. 82-88, 2002.

[6] R. Grisel, K. J. Weststrate, A. Gluhoi, and B. E. Nieuwenhuys, "Catalysis by gold nanoparticles," Gold Bulletin, vol. 35, no. 2, pp. 39-45, 2002.

[7] D. A. H. Cunningham, W. Vogel, R. M. Torres-Sanchez, K. Tanaka, and M. Haruta, "Structural analysis of $\mathrm{Au} / \mathrm{TiO}_{2}$ catalysts by Debye function analysis," Journal of Catalysis, vol. 183, no. 1, pp. 24-31, 1999.

[8] A. Ueda and M. Haruta, "Nitric oxide reduction with hydrogen, carbon monoxide, and hydrocarbons over gold catalysts," Gold Bulletin, vol. 32, no. 1, pp. 3-11, 1999.

[9] T. Herranz, X. Deng, A. Cabot et al., "Reactivity of Au nanoparticles supported over $\mathrm{SiO}_{2}$ and $\mathrm{TiO}_{2}$ studied by ambient pressure photoelectron spectroscopy," Catalysis Today, vol. 143, no. 1-2, pp. 158-166, 2009.

[10] Y. Xiao, F. Patolsky, E. Katz, J. F. Hainfeld, and I. Willner, "Plugging into enzymes: nanowiring of redox enzymes by a gold nanoparticle," Science, vol. 299, no. 5614, pp. 1877-1881, 2003.

[11] L. He, M. D. Musick, S. R. Nicewarner et al., "Colloidal Au-enhanced surface plasmon resonance for ultrasensitive detection of DNA hybridization," Journal of the American Chemical Society, vol. 122, no. 38, pp. 9071-9077, 2000.

[12] S. Andreescu and L. A. Luck, "Studies of the binding and signaling of surface-immobilized periplasmic glucose receptors on gold nanoparticles: a glucose biosensor application," Analytical Biochemistry, vol. 375, no. 2, pp. 282-290, 2008.

[13] C. R. Raj and B. K. Jena, "Efficient electrocatalytic oxidation of NADH at gold nanoparticles self-assembled on three-dimensional sol-gel network," Chemical Communications, no. 15, pp. 2005-2007, 2005.

[14] T. Liu, J. Tang, and L. Jiang, "The enhancement effect of gold nanoparticles as a surface modifier on DNA sensor sensitivity," Biochemical and Biophysical Research Communications, vol. 313, no. 1, pp. 3-7, 2004.

[15] C. Sönnichsen, B. M. Reinhard, J. Liphardt, and A. P. Alivisatos, "A molecular ruler based on plasmon coupling of single gold and silver nanoparticles," Nature Biotechnology, vol. 23, no. 6, pp. 741-745, 2005.

[16] G. C. Bond, "Gold: a relatively new catalyst," Gold Bulletin, vol. 34, no. 4, pp. 117-119, 2001.

[17] M. Valden, X. Lai, and D. W. Goodman, "Onset of catalytic activity of gold clusters on titania with the appearance of nonmetallic properties," Science, vol. 281, no. 5383, pp. 16471650, 1998.

[18] B. E. Salisbury, W. T. Wallace, and R. L. Whetten, "Lowtemperature activation of molecular oxygen by gold clusters: a stoichiometric process correlated to electron affinity," Chemical Physics, vol. 262, no. 1, pp. 131-141, 2000.

[19] L. Prati and G. Martra, "New gold catalysts for liquid phase oxidation," Gold Bulletin, vol. 32, no. 3, pp. 96-101, 1999.

[20] Y. Yuan, A. P. Kozlova, K. Asakura, H. Wan, K. Tsai, and Y. Iwasawa, "Supported Au catalysts prepared from $\mathrm{Au}$ phosphine complexes and as-precipitated metal hydroxides: characterization and low-temperature CO oxidation," Journal of Catalysis, vol. 170, no. 1, pp. 191-199, 1997.

[21] H. S. Oh, J. H. Yang, C. K. Costello et al., "Selective catalytic oxidation of CO: effect of chloride on supported Au catalysts," Journal of Catalysis, vol. 210, no. 2, pp. 375-386, 2002.

[22] Y. Li, H. J. Schluesener, and S. Xu, "Gold nanoparticle-based biosensors," Gold Bulletin, vol. 43, no. 1, pp. 29-41, 2010.
[23] C. Burda, X. Chen, R. Narayanan, and M. A. El-Sayed, "Chemistry and properties of nanocrystals of different shapes," Chemical Reviews, vol. 105, no. 4, pp. 1025-1102, 2005.

[24] N. L. Rosi and C. A. Mirkin, "Nanostructures in biodiagnostics," Chemical Reviews, vol. 105, no. 4, pp. 1547-1562, 2005.

[25] M. M. Alvarez, J. T. Khoury, T. G. Schaaff, M. N. Shafigullin, I. Vezmar, and R. L. Whetten, "Optical absorption spectra of nanocrystal gold molecules," Journal of Physical Chemistry B, vol. 101, no. 19, pp. 3706-3712, 1997.

[26] K. Rahme, F. Gauffre, J. D. Marty, B. Payré, and C. Mingotaud, "A systematic study of the stabilization in water of gold nanoparticles by poly(ethylene oxide)-poly(propylene oxide)poly(ethylene oxide) triblock copolymers," Journal of Physical Chemistry C, vol. 111, no. 20, pp. 7273-7279, 2007.

[27] C. Xu, X. Xu, J. Su, and Y. Ding, "Research on unsupported nanoporous gold catalyst for CO oxidation," Journal of Catalysis, vol. 252, no. 2, pp. 243-248, 2007.

[28] J. Kim, E. Samano, and B. E. Koel, "Oxygen adsorption and oxidation reactions on $\mathrm{Au}(2 \mathrm{l} 1$ ) $)$ surfaces: exposures using $\mathrm{O}_{2}$ at high pressures and ozone $\left(\mathrm{O}_{3}\right)$ in UHV," Surface Science, vol. 600, no. 19, pp. 4622-4632, 2006.

[29] V. Zielasek, "Gold catalysts: nanoporous gold foams," Angewandte Chemie, vol. 45, no. 48, pp. 8241-8244, 2006.

[30] M. M. Biener, J. Biener, and C. M. Friend, "Enhanced transient reactivity of an O-sputtered $\mathrm{Au}\left(\begin{array}{lll}1 & 1 & 1\end{array}\right)$ surface," Surface Science, vol. 590, no. 2-3, pp. L259-L265, 2005.

[31] N. R. Sieb, N. C. Wu, E. Majidi, R. Kukreja, N. R. Branda, and B. D. Gates, "Hollow metal nanorods with tunable dimensions, porosity, and photonic properties," ACS Nano, vol. 3, no. 6, pp. 1365-1372, 2009.

[32] M. Mohl, A. Jumar, A. L. Reddy et al., "Synthesis of catalytic porous metallic nanorods by galvanic exchange reaction," Journal of Physical Chemistry C, vol. 114, no. 1, pp. 389-393, 2010.

[33] C. Ji and P. C. Searson, "Fabrication of nanoporous gold nanowires," Applied Physics Letters, vol. 81, no. 23, pp. 44374439, 2002.

[34] J. Burdick, E. Alonas, H. C. Huang, K. Rege, and J. Wang, "High-throughput templated multisegment synthesis of gold nanowires and nanorods," Nanotechnology, vol. 20, no. 6, Article ID 065306, 6 pages, 2009.

[35] S. H. Yoo and S. Park, "Platinum-coated, nanoporous gold nanorod arrays: synthesis and characterization," Advanced Materials, vol. 19, no. 12, pp. 1612-1615, 2007.

[36] Z. Liu and P. C. Searson, "Single nanoporous gold nanowire sensors," Journal of Physical Chemistry B, vol. 110, no. 9, pp. 4318-4322, 2006.

[37] C. Ji and P. C. Searson, "Synthesis and characterization of nanoporous gold nanowires," Journal of Physical Chemistry B, vol. 107, no. 19, pp. 4494-4499, 2003.

[38] C. Ji, G. Oskam, Y. Ding, J. D. Erlebacher, A. J. Wagner, and P. C. Searson, "Deposition of $\mathrm{Au}_{x} \mathrm{Ag}_{1-x} / \mathrm{Au}_{y} \mathrm{Ag}_{1-y}$ multilayers and multisegment nanowires," Journal of the Electrochemical Society, vol. 150, no. 8, pp. C523-C528, 2003.

[39] L. Liu, W. Lee, Z. Huang, R. Scholz, and U. Gösele, "Fabrication and characterization of a flow-through nanoporous gold nanowire/AAO composite membrane," Nanotechnology, vol. 19, no. 33, Article ID 335604, 6 pages, 2008.

[40] S. R. Nicewarner-Peña, R. G. Freeman, B. D. Reiss et al., "Submicrometer metallic barcodes," Science, vol. 294, no. 5540, pp. 137-141, 2001.

[41] B. R. Martin, D. J. Dermody, B. D. Reiss et al., "Orthogonal self-assembly on colloidal gold-platinum nanorods," Advanced Materials, vol. 11, no. 12, pp. 1021-1025, 1999. 
[42] G. E. Possin, "A method for forming very small diameter wires," Review of Scientific Instruments, vol. 41, no. 5, pp. 772774, 1970.

[43] W. D. Williams and N. Giordano, "Fabrication of $80 \AA$ metal wires," Review of Scientific Instruments, vol. 55, no. 3, pp. 410 412, 1984.

[44] J. T. Masden and N. Giordano, "Localization and electronelectron interaction effects in thin Pt wires," Physical Review $B$, vol. 31, no. 10, pp. 6395-6401, 1985.

[45] R. M. Penner and C. R. Martin, "Preparation and electrochemical characterization of ultramicroelectrode ensembles," Analytical, vol. 59, no. 21, pp. 2625-2630, 1987.

[46] C. R. Martin, "Template synthesis of polymeric and metal microtubules," Advanced Materials, vol. 3, no. 9, pp. 457-459, 1991.

[47] C. K. Preston and M. Moskovits, "Optical characterization of anodic aluminum oxide films containing electrochemically deposited metal particles. 1. Gold in phosphoric acid anodic aluminum oxide films," Journal of Physical Chemistry, vol. 97, no. 32, pp. 8495-8503, 1993.

[48] D. Al-Mawlawi, C. Z. Liu, and M. Moskovits, "Nanowires formed in anodic oxide nanotemplates," Journal of Materials Research, vol. 9, no. 4, pp. 1014-1018, 1994.

[49] J. Erlebacher, M. J. Aziz, A. Karma, N. Dimitrov, and K. Sieradzki, "Evolution of nanoporosity in dealloying," Nature, vol. 410, no. 6827, pp. 450-453, 2001.

[50] J. Erlebacher, "An atomistic description of dealloying porosity evolution, the critical potential, and rate-limiting behavior," Journal of the Electrochemical Society, vol. 151, no. 10, pp. C614-C626, 2004.

[51] H. W. Pickering and C. Wagner, "Electrolytic dissolution of binary alloys containing a noble metal," Journal of the Electrochemical Society, vol. 114, no. 7, pp. 698-706, 1967.

[52] Z. Gu, H. Ye, D. Smirnova, D. Small, and D. H. Gracias, "Reflow and electrical characteristics of nanoscale solder," Small, vol. 2, no. 2, pp. 225-229, 2006.

[53] C. Schönenberger, B. M. I. van der Zande, L. G. J. Fokkink et al., "Template synthesis of nanowires in porous polycarbonate membranes: electrochemistry and morphology," Journal of Physical Chemistry B, vol. 101, no. 28, pp. 5497-5505, 1997.

[54] M. Tian, J. Wang, J. Kurtz, T. E. Mallouk, and M. H. W. Chan, "Electrochemical growth of single-crystal metal nanowires via a two-dimensional nucleation and growth mechanism," Nano Letters, vol. 3, no. 7, pp. 919-923, 2003.

[55] P. Y. Apel, I. V. Blonskaya, S. N. Dmitriev, O. L. Orelovitch, and B. Sartowska, "Structure of polycarbonate track-etch membranes: origin of the "paradoxical" pore shape," Journal of Membrane Science, vol. 282, no. 1-2, pp. 393-400, 2006.

[56] C. R. Martin, "Nanomaterials: a membrane-based synthetic approach," Science, vol. 266, no. 5193, pp. 1961-1966, 1994.

[57] T. M. Whitney, J. S. Jiang, P. C. Searson, and C. L. Chien, "Fabrication and magnetic properties of arrays of metallic nanowires," Science, vol. 261, no. 5126, pp. 1316-1319, 1993.

[58] C. J. Brumlik and C. R. Martin, "Template synthesis of metal microtubules," Journal of the American Chemical Society, vol. 113, no. 8, pp. 3174-3175, 1991.

[59] C. J. Brumlik, V. P. Menon, and C. R. Martin, “Template synthesis of metal microtubule ensembles utilizing chemical, electrochemical, and vacuum deposition techniques," Journal of Materials Research, vol. 9, no. 5, pp. 1174-1183, 1994.

[60] C. A. Foss, M. J. Tierney, and C. R. Martin, "Template synthesis of infrared-transparent metal microcylinders: comparison of optical properties with the predictions of effective medium theory," Journal of Physical Chemistry, vol. 96, no. 22, pp. 9001-9007, 1992.

[61] H. W. Wang, C. F. Shieh, H. Y. Chen, W. C. Shiu, B. Russo, and G. Cao, "Standing [111] gold nanotube to nanorod arrays via template growth," Nanotechnology, vol. 17, no. 10, pp. 2689 2694, 2006.

[62] S. Kumar, R. Kumar, S. Kumar, and S. K. Chakarvarti, "Electrochemical synthesis of metallic microstructures using etched ion tracks in nuclear track filters," Current Science, vol. 87, no. 5, pp. 642-645, 2004.

[63] R. K. Pandey, S. N. Sahu, and S. Chandra, Handbook of Semiconductor Electrodeposition, CRC Press, New York, NY, USA, 1996.

[64] A. Ertan, S. N. Tewari, and O. Talu, "Electrodeposition of nickel nanowires and nanotubes using various templates," Journal of Experimental Nanoscience, vol. 3, no. 4, pp. 287-295, 2008.

[65] A. Ertan, S. N. Tewari, and O. Talu, "Electrodeposition of nickel nanowires and nanotubes using various templates," Journal of Experimental Nanoscience, vol. 3, no. 4, pp. 287-295, 2008.

[66] D. F. Schriver and P. W. Atkins, Inorganic Chemistry, Oxford University Press, Oxford, UK, 3rd edition, 1999.

[67] T. C. Kuo, L. A. Sloan, J. V. Sweedler, and P. W. Bohn, "Manipulating molecular transport through nanoporous membranes by control of electrokinetic flow: effect of surface charge density and Debye length," Langmuir, vol. 17, no. 20, pp. 62986303, 2001.

[68] E. Seker, M. L. Reed, and M. R. Begley, "Nanoporous gold: fabrication, characterization, and applications," Materials, vol. 2, no. 4, pp. 2188-2215, 2009.

[69] M. Hakamada and M. Mabuchi, "Microstructural evolution in nanoporous gold by thermal and acid treatments," Materials Letters, vol. 62, no. 3, pp. 483-486, 2008. 

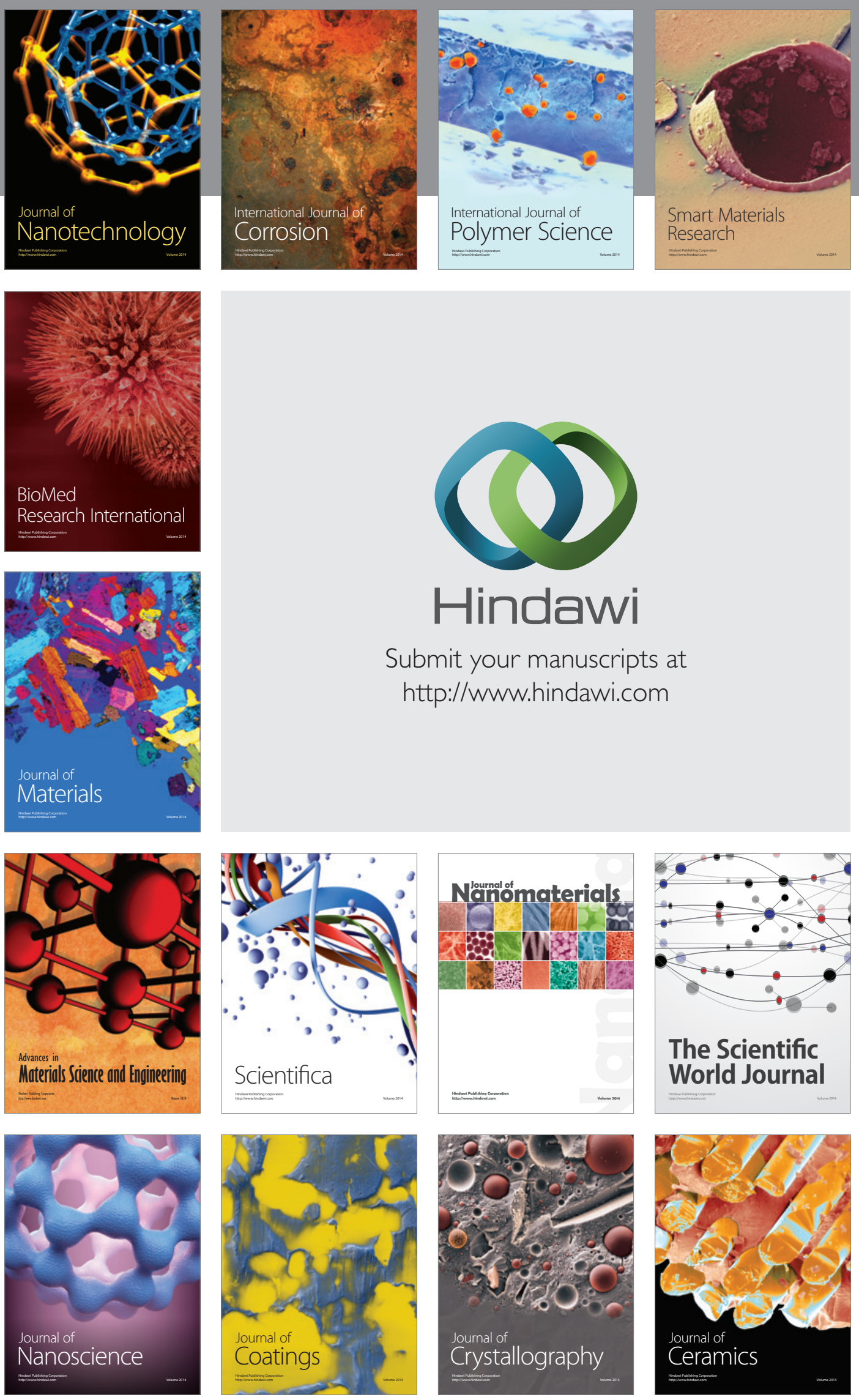

The Scientific World Journal

Submit your manuscripts at

http://www.hindawi.com

\section{World Journal}

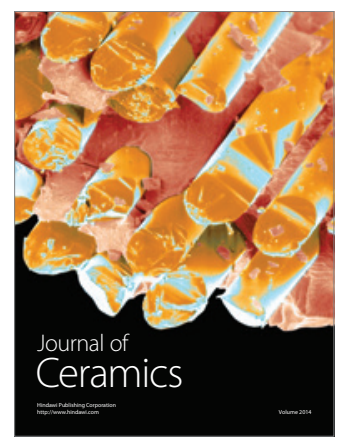

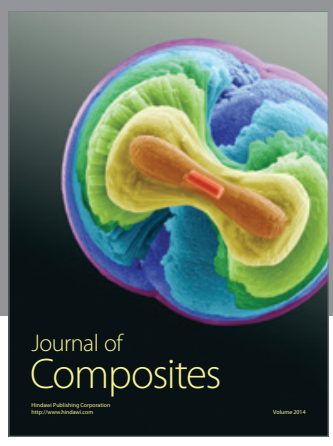
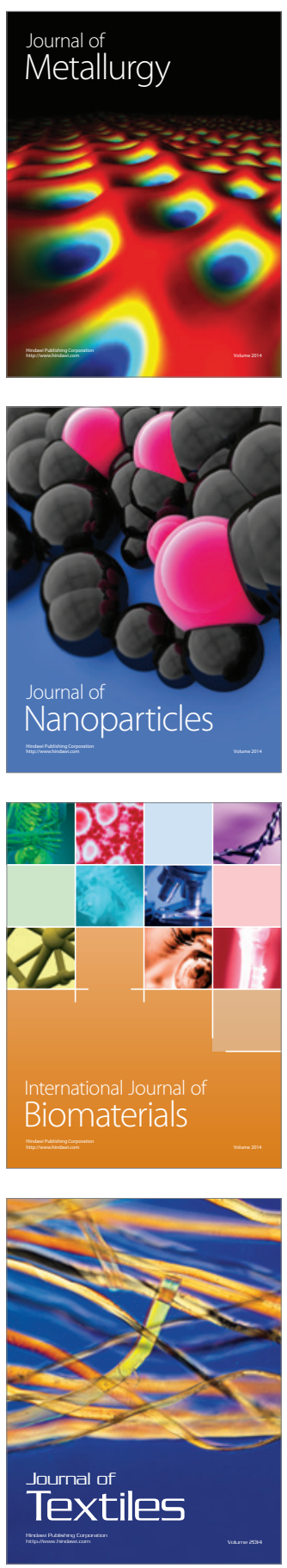